\title{
Gravitational Networks: self-organizing network topologies into hierarchically structured domains
}

\author{
Martin Johnsson and Brendan Jennings \\ Telecommunications Software \& Systems Group, \\ Waterford Institute of Technology, Ireland. \\ Email: mjohnsson@tssg.org, bjennings@ieee.org.
}

\begin{abstract}
Algorithms for self-organizing networks into node clusters have up to this point been limited to the organization of basically homogeneous networks of a limited size and application, having been targeted mainly towards wireless ad-hoc networks and sensor networks. We present a new distributed technique for how heterogeneous networks at any size, topology, and technologies can be self-organized into a set of domains using a novel method based on finding a centre node. The expression of finding the centre node is inspired by the law of finding the centre of gravity where the crucial problem sits in finding the node around which other nodes would "gravitate" and around which a domain can be formed. In this paper, we present the core principles and features of the GRANET concept, as well as presenting initial results from simulations of GRANET where its ability to scale and converge have been studied.
\end{abstract}

\section{INTRODUCTION}

Networks being able to self-organise themselves is by no way a new concept. It stems from needs of relaxing the requirements of human intervention and the human resources needed to configure network nodes in order to impose and control a certain organisation and structure of the network topology and its resources, e.g. to faciliate how routing tables are being organised and how routing information can be aggregated. The growing complexity, heterogeneity and the ever evolving requirement to support new types of applications and/or new traffic patterns, mean this task becomes untenable. The need for self-organisation is clear for many emerging networking scenarios, including large scale data centre interconnection, management of the deployment of small cell access networks, and the Internet of Things. As the Future Internet evolves it is clear that self-organisation, and self-management in general will be necessary and that such techniques will need to work on a global scale and be interoperable across different domains of control.

Ideally a self-organizing capability for networks should be highly scalable, be able to both respect as well to work across different domains, support the fact that networks can be instantly updated through addition or removal of nodes and links, and cope with heterogeneous network environments that may include both fixed and wireless network technologies as well as a huge spectrum of node types spanning from sensors up to core routers. As it should be able to operate in a huge variety of network enviroments, including networks which need to operate under specific policy settings, the mechanism should be highly configurable. The approach presented in this paper is based on the formula of finding the centre of gravity. We show how we can adapt this formulation to a networking context as the basis of a suitable means for network selforganisation. By identifying the nodes in a network topology which are located at centres for a distributed mass, we also find a feasible approach to domain formation as this centre node would be suitably located for a near optimal organisation of an arbitrary network topology. In this paper we present the concept of Gravitational Networks (GRANET) and its core, basic features, including a first stage of evaluations of its performance and characteristics.

\section{RELATED WORK}

Most work on clustering of nodes in a network has focussed on wireless ad-hoc networks and (wireless) sensor networks. Researchers thus generally assume a homogeneous network environment where nodes and links in the topology have the same order of capabilities and capacity; however, they do generally take into account that the network topology may change. The general problem to be solved is to define a mechanism/algorithm/protocol which can sub-divide a network into a set of node clusters, and where clustering needs to adapt to a changing network topology. This generally points to the issue of identyfing a Cluster Head $(\mathrm{CH})$ around which a cluster of nodes is created. The interesting questions are a) then which criteria are used for $\mathrm{CH}$ selection, b) how clustering is done, c) how both $\mathrm{CH}$ and clustering may adapt due to changing network topology, d) what is the convergence time for (re-)clustering, e) what cluster sizes, f) and how scalability aspects are supported? In the literature we see a huge range of approaches to answering these questions [1], [2], [3], [4], [5], [6], [8], [9], [11], [12], [13], [14]. These solutions are invariably targeted towards a very specific problem, with each making assumptions or operating within constraints that do not render them natural candidates for solution of the generalized self-organisation problem we address. We also pay attention to [10] which provides for a set of evaluation criteria such a proper number of clusters, cluster size distribution, and the proper number of levels for building a hierarchy of clusters.

\section{GRANET AT A CONCEPTUAL LEVEL}

In this section we present the main idea and concept behind GRANET as well as introducing the terminology used. 


\section{A. Candidate Node, Centre Node and Centroid value}

The name GRANET refers to "gravitational networks". At the centre of a body of distributed mass the torque exerted by the force of gravity evens out. If to translate this into a network context, we would argue that the centre would be located at the point where 1) the degree of connectivity as measured on the topology as a whole through the complete topology is highest possible, and 2) there is a minimum number of hops to reach all nodes in the topology. In addition, and once again inspired by the centre of gravity, the balance point is offset if the distributed mass has uneven density. Thus, the centre of the topology should take into consideration the "weight" of nodes as well as the distance of links. This leads to the expression below that defines the Centroid value for a node, which synthesizes the influence of the weight of a node, its topology-wide node degree, as well as its position in the network topology:

$$
\frac{\sum_{k=1}^{K} n_{w} \cdot d_{n}}{N^{2}}
$$

(Centroid)

Thus, the node which has the lowest Centroid Value will become a Centre Node. The Centre Node is chosen among the nodes having a Candidate Node status. Generally all nodes in the topology can be a Candidate Node, but it may also be a selected set of nodes (though all nodes in the topology are used in the calculation of the Centroid value), which provides support for that only authorized nodes shall be able to become Centre Nodes. $\mathrm{k}$ denotes the tier number, where a tier makes up all nodes at distance $\mathrm{k}$ hops from the node $(k=0$ denotes the tier where the node itself calculating its Centroid value is located, i.e. tier 0 ). $n_{w}$ denotes the weight of a node being in some tier $k$, and $d_{n}$ denotes the distance to that node. $N$ is the total number of nodes in the topology, and this normalization aspect of the Centroid value will be further dealt with below. As mentioned, the expression both takes the weight of nodes, node degree across all tiers, as well as the position in the topology into consideration through which we strike a balance between both these aspects into the equation of identifying Centre Nodes.

For the definition and calculation of weight and distance, this paper does not prescribe any specific syntax and semantics. Both can be a matter of explicit configuration, or take on some default values. However, we present one simple approach that can be used in calculations of the Centroid value. With regard to the weight, the power (in watts) of the node can be used as a metric. Chatterjee et al. [6] also look at weighting of nodes and mention a few other parameters that could be of interest if to refine and have a more elaborate calulation of a node's weight. With respect to distance we could use $d_{n}=$ propagationDelay/bandwidth. This calculation of distance both considers the actual physical length of a link as can be deduced from the propagation delay, and the "thickness" of the link as expressed by the bandwidth. Thus a longer link will have a higher value on propagation delay, and a link with lesser bandwidth will calculate a higher distance value. The calculatation of a distance between a Candidate Node and any other node, will need to add up the distance for each link that is traversed between the Candidate Node and the node for which the distance is to be calculated.

\section{B. Gravity Horizon and domain formation}

Through the expressions defined above we are thus able to identify the Centre Node of a network topology around which other nodes would "gravitate". The ability to gravitate is implied both through the weight of the Centre Node and its position. This means that we deem the fact that a node is selected as a Centre Node provides substance for being able to attract other nodes, both through its position in the network, which generally means it will be able to attract more nodes than a node in the periphery, as well as its weight, which points to a processing capability of handling relatively more nodes. Further, as the force of gravity is inversely proportional to the distance, a Centre Node is only able to attract nodes being within what we term the Gravity Horizon. The Gravity Horizon is supported by a maximum and minimum distances for the Gravity Horizon; the GH Max Distance and the GH Min Distance. They are both expressed as a hop distance from the Centre Node. These max and min values are also used to ensure a certain level of fairness between Centre Nodes when they attract surrounding nodes. There is also a need to have decision logic to control where a node shall belong to in case a node is attracted to two or more Centre Nodes when and if they may fall within the distance of multiple Gravity Horizons. The following arbitration logic could then be used (subject to potential refinement depending on preferences):

- Centre Node with lowest Centroid value;

- If equal, it shall choose the one at the shortest distance;

- If equal, it shall choose the one with highest node weight;

- If equal, it shall choose the one with highest node identity.

From this it follows that a Centre Node can form a Domain consisting of itself and all the nodes which falls within its Gravity Horizon in accordance with these rules. We can then express the Domain Node Set consisting of:

- the Centre Node,

- All nodes within GH Min Distance,

- All nodes within the GH Max Distance, except the nodes for which there is an overlapping Gravity Horizon with one or more other Centre Nodes and where those other Centre Nodes wins a node according to above logic.

The Domain Node Set is key to maintin an up-to-date record of each node's belonging and further provides support for e.g. routing, name resolution, and information management applications.

\section{Topology Horizon}

The distributed processes of topology discovery and domain formation are generally independent in GRANET. If the optimal Centre Node is to be found, i.e. the one with the minimum Centroid value, the calculation should be done over the entire topology. This is generally not feasible for any 


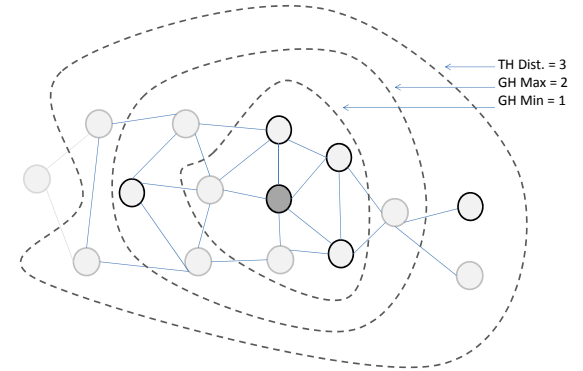

Fig. 1. An illustration of the relationship between the Gravity Horizon (GH Max and GH Min Distance, and the Topology Horizon(TH Distance) Ordinary nodes are unfilled, Candidate Nodes have a filled outer rings, and Centre Nodes have both filled the ring and the inner circle filled.

mechanism that are to operate on a very large or even global scale. For this purpose, GRANET introduces the concept of a Topology Horizon, which in the case of GRANET is the part of the complete network topology that a particular node can see. The Topology Horizon is defined as the topology being visible within a certain number of hops from any given node. Thus, topology discovery is confined to operate within the $\mathrm{TH}$ Distance.

This leads to a sub-optimal selection of the Centre Node as it will be based on a) an incomplete network topology, and b) the fact that each node will compute a Centroid value based on different incomplete views of the network topology. This suboptimal calculation of the Centroid value is however of less concern to the GRANET mechanism as GRANET through the Centroid value calculation gives priority to nodes with a high degree of connectivity closer to a node, as well as the fact that GRANET has not as a prime focus to find the Centre Node for the complete topology but rather local Centre Nodes that can serve as a centre for a domain within its "local neighborhood". Now going back to the formula for calculating the Centroid value, the normalization with $N^{2}$ is needed as a well-connected node more centrally positioned will likely have far more total neighbors within its Topology Horizon compared to a node in the periphery of the topology, and this number tends to grow exponentially rather than proportionally.

Figure 1 above illustrates how the different terms described above relates to each other and how they are applied to form a Domain.

\section{Recursion and resilience}

The GRANET concept supports recursion. This means that a Centre Node of a Domain is able to instantiate a Supernode that will represent the Domain at the next level of recursion. The Supernode will represent the capacity of all the nodes and links in the underlying Domain. A simple approach to calculate the weight for the Supernode is to add up the weights for each of the underlying nodes. To represent links, there is a need to aggregate the underlying links for representation at the next domain as a Link Abstraction. A Link Abstraction shall properly represent the distance of the underlying links it represents. A simple approach to calculate an aggregated distance for parallel links is to a) select the highest propagation delay among the parallel links, and b) summarize the bandwidth for all the links.

The Centre Node's only role in GRANET is to maintain the Supernode. If it fails, any other Candidate Node in the Domain and listed in the Domain Node Set may become the new Centre Node, and could take over the role and represent the Domain through the very same Supernode.

\section{E. Domain levels and domain borders}

Each node and each node interface could be pre-configured with a Domain Level, or provided by an "upstream" network, or alternatively could be deduced from for example the node's weight. As a general rule, each node interface will first be given a Domain Level that equals the node's Domain Level. If this node interface will become a domain border towards another Domain due to domain formation, that node interface will be given a new value that corresponds to the next higher level in the domain hierarchy. This type of Domain Level configuration of node interfaces are referred to as "soft domain borders" as they are induced by GRANET and the selforganisation of the network topology and may later change when and if the topology changes.

In case a specific node interface is explicitly configured with a Domain Level, that interface becomes a "hard domain border", which basically stops all protocol interactions across that interface up to the point indicated by the Domain Level.

\section{F. Domain sizes}

GRANET generally operates out from the Gravity Horizon when forming Domains. The connectivity degree will then implicitly define the size of a formed domain, the higher the degree the bigger the domain. GRANET allows configuration of both maximum and minimum sizes of domains.

\section{G. Example network}

Figure 2 below shows an example network topology indicating each node's weight and the distance of each link. Only the Candidate Nodes have been given names. The Topology Horizon is 4, and the GH Max/Min Distances are 2 and 1 respectively. This results in a Domain configuration as indicated by the dotted curves in Figure 3 below. A few comments:

- It is the $\mathrm{m}, \mathrm{d}$, v Candidate Nodes who can become Centre Nodes and form domains without competition, i.e. their respective Centroid value is the lowest within their respective Gravity Horizon. At that stage they select all nodes within their respective GH Max Distance (this stage is not depicted). - The $r$ and $f$ Candidate Nodes can become Centre Nodes first after the $\mathrm{m}$ and $\mathrm{d}$ domains have been formed. This is due to that $\mathrm{m}$ took both the $\mathrm{h}$ and $\mathrm{g}$ nodes into its domain, and $\mathrm{d}$ took the e and c nodes. This removed the competition from $r$ and $\mathrm{f}$ who is then able to become Centre Nodes. $\mathrm{r}$ will reclaim the ordinary node above the $\mathrm{h}$ node from $\mathrm{m}$ as that node is within the GH Min Dist. of r. f will reclaim the g node and the ordinary node left of the $\mathrm{g}$ node as they are within the GH Min Dist. of $f$. 


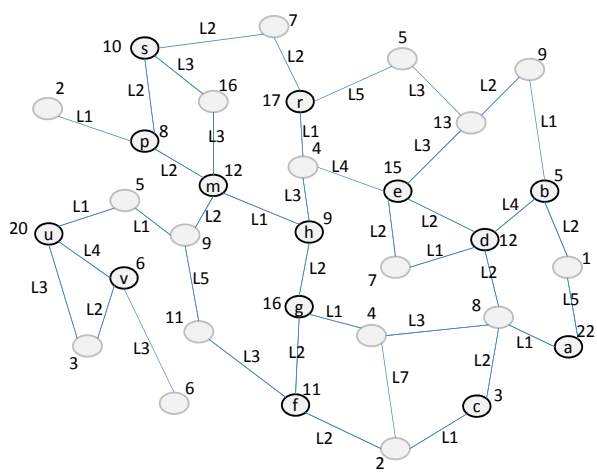

Fig. 2. An example network topology, each node's weight and the distance of each link (preceeded with an "L" for clarity) are depicted.

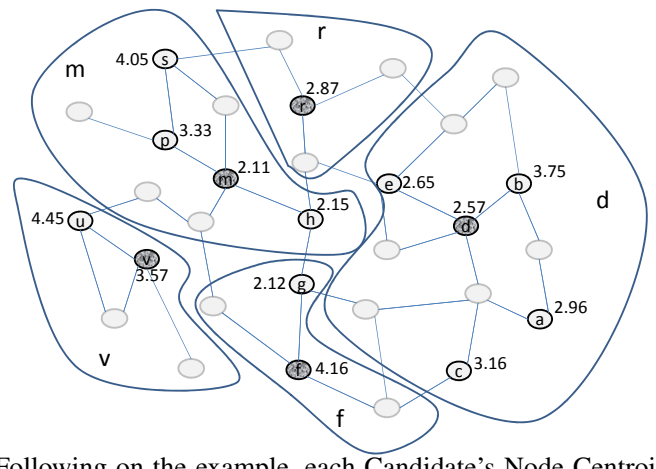

Fig. 3. Following on the example, each Candidate's Node Centroid value is here shown together with the end result of the Domain Formation process.

\section{SimUlATION RESULTS}

GRANET has been implemented in all new network simulator written in $\mathrm{C}++$ with the specific purpose to be a testbench for new algorithms that can be evaluated based on a completely generalized model of a network and without dependencies to existing technologies, more information can be found at [7]. Each iteration of the GRANET protocol consists of a cycle of topology discovery and domain formation. In our simulation study we focus on the following performance metrics:

Network bootstrapping We measure the time it takes to bootstrap a network as the time between the network topology has been created until the network is completely and fully self-organized into a set of domains, including the complete domain hierarchy with Supernodes and Link Abstractions. We call this time $T_{\text {boot }}$ and is measured as a number of iterations of the GRANET protocol.

Network re-convergence We measure the time it takes to re-converge a network as the time between GRANET's first observation of the event (e.g. a node goes down), and the time when all the domains have completed their domain formation cycle to completeness. We call this time $T_{r c v}$ and is also measured as a number of iterations of the GRANET protocol.

Domain stability The Domain Stability metric DoSt is measured as a relative difference to the Domain Node Set before, $D N S_{\text {old }}$, and after the event (a sum of new nodes having entered into the Domain, $D N S_{n e}$, and nodes having
TABLE I

SIMULATION RESULTS.

\begin{tabular}{llll}
\hline Metric & 10 nodes & 70 nodes & 300 nodes \\
\hline Mean domain size & 7.2 & 7.8 & 8.1 \\
Min domain size & 3 & 4 & 4 \\
Max domain size & 10 & 12 & 15 \\
Mean No Domains & 1.3 & 9.5 & 36.7 \\
$T_{\text {boot }}^{-}$ & 3.4 & 17.7 & 19.2 \\
$T_{r c v}^{-c v}$ & 3.9 & 3.8 & 3.8 \\
$D^{-} S t$ & $0.45(2)$ & $0.19(2.1)$ & $0.14(1.9)$ \\
\hline
\end{tabular}

left the Domain, $D N S_{n l}$ ), i.e.:

$$
D o S t=\frac{D N S_{n e}+D N S_{n l}}{D N S_{\text {old }}}
$$

DoSt shall be as close to 0 as possible. DöSt is calculated for those Domains that encountered any change at all in their Domain Node Set and that mean number of Domains are provided in paranthesis.

\section{A. Results}

Each of these metrics have been evaluated using an arbitrary network topology consisting of bilateral links and with an average node degree of 1.5 at the edge and 3 towards the topology centre, and with topologies at the size of 10, 70, and 300 nodes. GRANET was configured with a GH Max/Min Dist $=2 / 1$, TH Dist $=5$, all protocol timers fixed however jittered to avoid a completely synchronized transmission of messages, node weights and link distances all set to 1 , min domain sizew set to 3 (no upper bound on max domain size) and all nodes are Candidate Nodes.

The results together with some additional stats are provided in Table 1 below. The result is affected by the fact that a newly elected Centre Node needs to keep its status for 3 consecutive iterations before it is allowed to instantiate a Supernode. The table also depicts results after a domain being smaller than the minumum domain size has been merged into another domain.

\section{CONClusion}

We have in this paper presented a new mechanism aimed for self-organisation of networks at very large scale called GRANET. Initial results from simulations show that GRANET is performing according to its definition and expectations, however more elaborate and larger scale simulations are needed and more metrics are also needed, e.g. a metric on looking at domain border stability. We're also looking at finding further abilities for GRANET to self-configure, e.g. to find out the optimum values on GH Max/Min Distances at each domain level.

\section{ACKNOWLEDGEMENT}

This work has been funded by Science Foundation Ireland, via the "FAME" Strategic Research Cluster, grant no. 08/SRC/I1403. 


\section{REFERENCES}

[1] A. D. Amis, R. Prakash, T. H. P. Vuong, and D. T. Huynh. "Max-Min D-Cluster Formation in Wireless Ad-hoc Networks", Proceedings of 19th Annual Joint Conference of the IEEE Computer and Communications Societies (INFOCOM 2000), IEEE, 2000, vol. 1, pp. 32-41.

[2] D. J. Baker and A. Ephremides, "The Architectural Organization of a Mobile Radio Network via a Distributed Algorithm", IEEE Transactions on Communications, IEEE, 1981, vol. 29, no. 11, pp. 1694-1701.

[3] S. Banerjee and S. Khuller, "A Clustering Scheme for Hierarchical Control in Multi-hop Wireless Networks", Proceedings of the 20th Annual Joint Conference of the IEEE Computer and Communications Societies (INFOCOM 2001), IEEE, 2001, vol. 2, pp. 1028-1037.

[4] S. Basagni, "Distributed Clustering for Ad-hoc Networks", Proceedings of the 4th International Symposium on Parallel Architectures, Algorithms, and Networks (I-SPAN 1999), IEEE, 1999, pp. 310-315.

[5] S. Basagni, "Distributed and Mobility-adaptive Clustering for Multimedia Support in Multi-hop Wireless Networks", Proceedings of the IEEE VTS 50th Vehicular Technology Conference (VTC 1999), IEEE, 1999, vol. 2, pp. $889-893$.

[6] M. Chatterjee, S. K. Das, and D. Turgut, "WCA: A Weighted Clustering Algorithm for Mobile Ad Hoc Networks", Cluster Computing, 2002, vol. 5, no. 2 , pp. $193-204$

[7] FlatEarth Simulator. [Online] Available (13/5/13): http://github.com/koldemo/FlatEarth.git

[8] P. Krishna, M. Chatterjee, N. H. Vaidya, and D. K. Pradhan, "A clusterbased approach for routing in dynamic networks", ACM SIGCOMM Computer Communication Review, ACM, 1997, vol. 27, no. 2, pp. 49-64.

[9] R. Krishnan and D. Starobinski. "Efficient clustering algorithms for selforganizing wireless sensor networks." Ad Hoc Networks, vol. 4, no. 1, 2006, pp. 36-59.

[10] J. Lian, Jie, K. Naik, and G. B. Agnew, "A Framework for Evaluating the Performance of Cluster Algorithms for Hierarchical Networks", IEEE/ACM Transactions on Networking, IEEE/ACM, 2007, vol. 15, no. 6, pp. $1478-1489$

[11] C. R. Lin and M. Gerla, "Adaptive Clustering for Mobile Wireless Networks", Journal on Selected Areas in Communications, IEEE, 1997, vol. 15 , no. 7 , pp. $1265-1275$.

[12] A. B. McDonald and T. F. Znati. "Design and Performance of a Distributed Dynamic Clustering Algorithm for Ad-hoc Networks, Proceedings of the 24th Annual In Simulation Symposium, IEEE, 2001, pp. 27-35.

[13] A. K. Parekh, "Selecting Routers in Ad-hoc Wireless Networks", In Proceedings SBT/IEEE Intl Telecommunications Symposium, SBT/IEEE, 1994, pp. 420-424.

[14] O. Younis and S. Fahmy, "HEED: a hybrid, energy-efficient, distributed clustering approach for ad hoc sensor networks", Transactions on Mobile Computing, IEEE, 2004, vol. 3, no. 4, pp. 366-379. 